\title{
The S-Word is Taboo: Shame is Invisible in Modern Societies*
}

Thomas Scheff ${ }^{* 1}$ Steve Mateo ${ }^{2}$

1Department of Sociology, UCSB, University of California, 3009 Lomita Road, Santa Barbara CA, USA

${ }^{2}$ Child Care Worker, Trinity Group Homes Inc

*Corresponding author: Thomas Scheff, Department of Health Services, University of California, 3009 Lomita Road, Santa Barbara CA, USA, Tel: (310) 513-2715; Email:xscheff@gmail.com

Rec date: Jan 02, 2016, Acc date: Jan 4, 2016, Pub date: Jan 12, 2016

Copyright: ( 2016 Scheff T. This is an open-access article distributed under the terms of the Creative Commons Attribution License, which permits unrestricted use, distribution, and reproduction in any medium, provided the original author and source are credited.

\section{Abstract}

Great is truth, but still greater...is silence about truth. ...simply not mentioning certain subjects... influences opinion much more effectively than ...the most eloquent denunciations. (Aldous Huxley).

This essay proposes that, like all other mammals, all human groups are built around shame. In the typical idea of shame in modern societies, it is seen as triggered by some particular cultural/personal stimulus (nakedness, for example). However, there is also a universal component, set in motion by threat to the social bond. If that is the case, we need to reclaim the many fields that so far have used alternate terms, such as honor, stigma, fear of rejection, disrespect, social pain, and so on. Although there is an actual literature on shame, it is dwarfed by the many studies that use other terms. The s-word, like the f-word, is usually taboo, both in the public and in publications. This essay describes at length five fields that hide their shame content, and also, more briefly, several more. The hiding of most shame studies is further evidence of how deeply shame is still taboo in modern societies.

The first studies of sexual behavior published $[1,2]$ met condemnation because they discussed subjects that had previously been taboo. Yet they quickly became known both to the research world and to the public at large. Suppose, however, that they had used an inoffensive but ambiguous word like LOVE or INTIMACY instead of the word SEX, which at that time was more taboo than it is now. They would have caused little offense, but their work would have become less well known. If that were the case, there would have been almost no effect on the public.

Studies of the emotion of shame still face a similar dilemma because shame, much more today than sex, is still quite taboo. Consider the case of a study of shame that avoided the s-word in the title, but discussed it fully in the text. The noted psychiatrist [3] contended that secret shame is the cause of violence, based on his experience as a prison psychiatrist. When he asked prisoners why they killed, the answers were virtually all the same: being dissed (disrespected). Unlike most researchers in his place, Gilligan didn't write a book about dissing or even disrespect as a cause of violence. Instead, he related it to what might turn out to be a universal human emotion, shame.

Although there is a personal and cultural part of shame, it also always has a universal mammalian component [4] as signal of threat to the social bond, the feeling, however slight or intense, of rejection. For example, traditional societies tolerate public nakedness in varying degrees, modern societies do not. But what is essential in both types of culture is that the individual see the forbidding as coming from his or her own group, signaling the possibility of rejection. The life of all mammals depends on acceptance from others, especially in early life, but also throughout life. Humans are no exceptions. The need for acceptance is so taken for granted that we forget that we are seeking it almost constantly.

*Steven Mateo helped me explore the literature of hidden shame.

As Charles Cooley, an early American sociologist wrote: "We live in the mind of others without knowing it." In order to understand spoken language, which if taken literally is quite ambiguous, children must learn at an early age to try to see what is said from the point of view of the speaker. As they become competent at role-taking, they learn it so rapidly and so often that they forget they are doing it. But this competence opens up a huge cavern of signals of possible rejection.

The last part of Cooley's sentence, "without knowing it," points to a serious difficulty in studying humans: they seem to be quite unaware of the vast amounts of time they spend in the minds of others, and therefore how much shame they undergo. A more specific cause of difficulty is that even more than the f-word, the s-word is usually taboo. One aspect of the taboo shows up clearly in academic research: there are many studies of the shame system, but hidden under other terms: fear of rejection, disrespect, stigma, honor cultures, revenge, etc. 
Gilligan's book has had only modest success commercially and academically. It was never on the bestseller lists; it stands currently around the 30 thousandth mark. According to Google Scholar, it has been cited 400 times, which is 24 times a year since its publication. For a open statement about the cause of all violence, it seems that it has receive only modest notice by the public or by scholars. If the word shame had appeared in the title, it might have fared much worse.

With a different title and approach, perhaps it would have been more popular. The actual title, Violence: reflections on a national epidemic, is not exciting or revealing of the content. The publisher may have not allowed the use of shame in the title, as sometimes happens [1]. Perhaps Dissing and Violence would have had more appeal. But if Gilligan had wanted to have the word dissing in the title, he might have had to stick with the dissingdisrespect thesis, not even mentioning his notion of secret shame. The s-word would be not appealing, even repulsive.

What could be repulsive about the s-word, since it's only a word? One could ask the same question about the fword, since it also is only a word. It is clear that the f-word was usually repulsive for the sixty years before 1961, at least in print. According to the Google Ngram, there was not a single occurrence of the word fuck in books in the English language between 1900 and 1960. It appears that printed books were fussy about this matter, since when I was in basic training in the Army in 1953, it was almost every other word out of the mouths of trainers and trainees alike.

Oddly, with the f-word getting more exposure beginning in 1961, the s-word has been getting less. The N-gram shows that the frequency of use in English language books has been decreasing steadily for two hundred years (1800-2000). To see if this decline was in English only, I checked the Ngrams for French (honte), German (schande) and Spanish (verguenza) equivalents. The decrease has been occurring in these languages also over the two hundred years. What is going on? [1] I know that this was the case [5, 6] book, which was entirely about the humiliations of the Third World by the First World. I learned that this book was about shame only by a chance meeting with him on a lecture tour in England.

\section{The Taboo on Shame}

From his study of five hundred years of European history, the sociologist [7] proposed that shame and its close kin (embarrassment and humiliation) have become the dominant emotions in modern societies, even though they are taboo. As already indicated, these three emotions have also been frequently studied in social/behavioral, political and medical science (particularly psychiatry), and history, but under different names.

There are many studies in anthropology of "cultures of honor": how insults to honor lead to humiliation and revenge. Most of these studies however, assume that this sequence causes violence only in traditional societies, where shame is out in the open. It is not considered to occur in modern societies. Although the word honor has gone out of style, the emotion of shame has not. If it is biologically based, it is also ahistorical and cross cultural.

The taboo on shame has many weakening effects on knowledge, because it cordons off into separate groups what ought to be a single field, reinforcing the existing taboo. For example, it hides other studies that support's Gilligan's conjecture on hidden shame as a cause of violence, such as status attainment, loss of social status, search for recognition, honor/dishonor, vengeance or revenge, and so on. It also slows down the process of replicating studies that support it $[8,9]$ and testing a broader hypothesis extending to causes of both violence and silence [10]. If the shame-violence/silence hypothesis is even partly true, it carries a crucial message for our civilization.

Elias's thesis, in more detail, is that there is a difference between shame that is felt, the basis of morality, and shame that is hidden not only from others but even from self. In his study he analyzed etiquette manuals in three languages. Two key findings [5], as physical punishment decreased, shame became dominant as the main agent of morality [11]. As shame became more prevalent, it also went underground, becoming virtually invisible.
How can shame become invisible? Modern audiences cannot accept this idea, since they conflate emotion and feeling. However, most people will agree that at times a person's anger can be obvious to others, yet the angry person completely unaware of it. A similar argument can be made about fear: since boys, especially, are taught to equate fear with cowardice, they learn to automatically suppress fear to the point that they don't feel it. It may be that recklessness, particularly, arises from this process. Similarly, perhaps a person can be in a bodily state of shame without feeling ashamed.

Elias interpreted invisibility in terms of taboo: in modernization shame becomes a topic that is not to be talked about, just as sex was such a topic in the 19th century. As sex and especially the f-word were taboo then, so the s-word has become taboo now. The psychologist [12] is one of several writers who have argued that shame is taboo in our society:

American society is a shame-based culture, but ...shame remains hidden. Since there is shame about shame, it remains under taboo. ....The taboo on shame is so strict that we behave as if shame does not exist [12].

In addition to Elias, another early discovery of the taboo on shame was made by Helen Lewis (1971), a research and clinical psychologist. She used the technique to locate emotion episodes in the transcriptions, and then analyzed the reactions of both client and therapist to each episode. The Gottschalk method concerns words and phrases that are commonly understood to indicate emotions, such as "being pissed off" as a way of referring to anger, or "feeling rejected" to shame. The device is now available as software, the (Picard 2000) [13].

Lewis's analysis of the results led to two surprises. First, shame episodes were by far the most frequent, outnumbering all the other emotions combined. Secondly, unlike the other episodes, such as anger. The shame episodes were not commented on. Neither therapist nor client seemed to notice them. 
In the book, she referred to the seemingly unnoticed shame as unacknowledged, since she couldn't tell whether the therapists and clients were unaware of the emotions, or whether they were aware but not mentioning them. Since Lewis was a practicing psychoanalyst as well as a researcher, she later questioned her own clients when they used words that indicate shame. She found them to be unaware of the shame that their wording implied.

In the 1971 book, in connection with the sequences that occurred after the client's shame episodes, Lewis made two further discoveries. The most frequent sequence was what seemed to be varying degrees of withdrawal by the client. Lewis called this a sequence from shame to depression. The client would begin to speak less and more slowly with less clarity. There was, however, also another response, a sequence from shame to anger, sometimes at the therapist. The anger reaction was less frequent than withdrawal. These two sequences may turn out to be quite important.

As indicated, shame episodes never led into discussion of the episode by client and/or therapist. For example, if something the therapist had said embarrassed the client, he or she might have responded with "That remark you just made me feel ashamed," or even the more indirect "You hurt my feelings." Such a statement might have then led the discussion toward working through the client's shame, a therapeutic sequence. Such a sequence did not occur in any of the 150 sessions.

\section{Hiding Shame}

Lewis proposed that shame is mostly hidden both from others and from self. She found that shame goes unacknowledged in two different ways. The first way she called "overt, undifferentiated shame" (OU). The client is in pain, but it is referred to indirectly, at best. As [13], there are hundreds of words and phrases in English that can be used to refer to shame without naming it. For example, one can say "I fear rejection," or "This is an awkward moment for me," and so on. Many of these cognates have been listed [14] OU shame is usually marked not only by pain, but often by confusion and bodily reactions: blushing, sweating, and/or rapid heartbeat. One may be at a loss for words, with fluster or disorganization of thought or behavior, as in states of embarrassment. Many of the common terms for painful feelings appear to refer to this type of shame, or combinations with anger: feeling hurt, peculiar, shy, bashful, awkward, funny, bothered, or miserable; in adolescent vernacular, being freaked, bummed, or weirded out. The phrases "I feel like a fool," or "a perfect idiot" is prototypic.

\section{Reclaiming Shame Studies}

Shame and its siblings are much less discussed than other emotions, not only by the public, but also researchers. How could that be? There have been many studies of shame, but most of them use what Elias called circumlocutions. An illustrative example is found in a recent study of doctor-patient relationships [15]. Instead of referring to how the doctor may shame a patient, the title uses the phrase "disrespectful behavior toward patients." The article makes no reference to shame. Although the reader will understand what is meant, the phrase cuts the authors and the readers off from an understanding of shame dynamics that are available in the literature on shame and its siblings.

Studies of the facial expression of emotion, a huge field, were based on the foundational descriptions of basic emotions [16]. But for many years until quite recently, these studies did not include shame, even though Tomkins gave more attention to it than any other emotion (V. II, 1963).

Another example is stigma. There are thousands of studies in the social, behavioral and medical sciences of this topic. The idea is that police arrest or illness diagnosis may carry with it an unintended consequence: the shaming of the recipient, to self and/or his/her social network. These studies virtually never use the term shame in the title, and in most cases, even in the body of the study. This case illustrates the taboo the most emphatically, since shame is the actual dictionary meaning of stigma.

Clearly equated stigma with shame [17] (pp. 13, 14, 25, 108). But by 1980 , when articles on stigma started appearing, the term shame had disappeared. This absence is particularly noticeable in an attempt to conceptualize stigma [18]. Although they quote Goffman's book at some length, there is no mention of his use of shame.

Another example: more than twenty thousand studies using selfesteem scales. They all may be making the same error of using a multivariate scale, one cognitive, the other emotional, that involves the pride/shame axis [19] that cancel each other out.

This essay used a systematic method to locate hidden shame studies. $[13,11]$ devised a method that finds alternate naming for the major emotions, such as anger ("hot under the collar"), grief, fear, and shame. We have used this method and the update [14], (Chapter 3) to locate a random sample of studies in the many fields that directly study shame, but use alternative names for it. The first example does use shame to analyze the material studied, and, the only one of the six; it also references the shame literature.

1. Adair, David. The Injuries of Hidden Class: Neoliberalism and the Crimes of Inequality." (2012): 57- 65.

Adair explains the neo-liberalistic turn as the main reason for the replacement of 'class' to 'inequality' during that time; moreover, the emphasis on the term 'inequality' since the neoliberal has been largely due to the reason of an increased gap between the rich and the poor. Mentioning the Marxist perspective, Adair mentions reasons inequalities of income and opportunities are built into the wage system of a capitalist system. Additionally, due to the marketing technique of introducing products higher in the social ladder which eventually trickle down to the lower levels, workers are caught in a cycle of trying to reach an income of living with the commodities of the social class above them. Adair makes the shame literature connection, in attempting to define shame, by stating shame, embarrassment and humiliation are more often used in the forms of marker words, such as injured dignity, self-doubt or feelings of powerlessness and the "ringmaster" of these hidden terms, of which suggest wider emotional and cognitive complexes, is in fact shame. From two narratives mentioned in the story, Adair states in his narrative of being told courteously to stop smoking in the factory where he worked, he felt ashamed thereafter although he did not know it yet and couldn't understand why he felt the way he did. Through contemplation and time did he come to discover what he felt at that moment was humiliation, a strong pain of shame in his chest, and recounted how he attempted to mask whatever he believed he was feeling at the time. Back to the main point, Adair declares, currently, the lower classes in the social hierarchy may in fact feel ashamed of themselves in their situation; thus, shame may bring about responses such as physical violence, addiction, depression and isolation. Successfully differentiating shame from embarrassment is just one of the many obstacles researchers need to overcome when looking into the field; Adair concludes by advising 
one possible solution to resist humiliation of class in the hierarchic workplace is to take pride in work, but nothing can be done from the stigma/shame brought about from one's lack of consumption due to their socio-economic status.

Word Count: Shame: 53

Embarrassment: 3

Humiliation: 11

Disrespect: 1

Stigma -3

Shame literature citations: $5<$ www.groterebermuseum.org.au>

The next five examples are much more typical: little or no use of shame terms and few if any citations from the shame literature.

Warren, Charlotte et al. "Study Protocol for Promoting Respectful Maternity Care Initiative to Assess, Measure and Design Interventions to Reduce Disrespect and Abuse during Childbirth in Kenya." BMC: Pregnancy and Childbirth. 24 Jan, 2013 www.biomedcentral.com

Due to financial and geographical barriers, access to skilled birth attendants is rare in developing countries, particularly in Sub-Saharan Africa; therefore, it comes as no surprise pregnancy and childbirth are the leading causes of death for women of reproductive age in these disadvantaged areas. As a result of inexperienced birth attendants, disrespect and abuse is common and may take several forms: physical abuse, non-consented care, non-confidential care, and non-dignified care, and discrimination, abandonment of care and detention of facilities. Additionally, little is being done about the issue though policy makers, program staff, civil society groups and community members are well aware of these behaviors. The study, seeking to ultimately reduce disrespectful and abusive behavior towards woman in labor, observed seven health care facilities in total and included several independent variables, which involved a client interview to measure their perceptions of working conditions, respect, empathy with and prejudice against clients, and awareness of policy and service delivery guidelines concerning respect, dignity and client rights. Although this study has not taken place yet, as it is only a protocol of proposed ideas, the proposal implies the many manifestations of disrespect and abuse, ranging from neglect of dignity to physical violence, create an strong enough impact in female clients that it may play a critical part in the birthing process, aside from limited staff and little knowledge of health rights, in health care facilities of developing countries.

Shame: 2 (Note: Both mentions of shame were from one title in the references section), Embarrassment: 0, Humiliation: 1, Disrespect: 29. No references to shame literature.

URL: http://www.biomedcentral.com/1471-2393/13/21/

Berkhout, Suze G. "Private Talk: Testimony, Evidence, and the Practice of Anonymization in Research." International Journal of Feminist Approaches to Bioethics. 6.1 (2013): 19-45.

Focusing on the problems research participants may have with anonymization, Berkhout claims removing any form of identity does little to address the politics of knowledge production. In her study, concerning socially and economically disadvantaged women navigating through the increasingly complex health-care system, Berkhout addressed the importance of confidentiality to her participants as she noted voluntarily choosing identifiable pseudonyms, such as nicknames, could lead to embarrassing, discrediting, or even discriminatory information; in other fields of research, the neglect of anonymity may also very well lead to a risk of discrimination through illness or disability disclosure. Berkhout further claims anonymity can also protect from harm of certain individuals, strengthen the researcher- participant relationship; however, in some cases, participants neglect anonymity since they feel the researcher would prefer a completely honest testimony. Though this analysis centralizes on the problems participants may have with anonymity, Berkhout emphasizes the importance of anonymity to her participants since the lack of, depending on the context, could easily lead to embarrassment, or shaming, of the participants through disclosed information leaking out in the open.

Shame: 0, Embarrassment: 3, Humiliation: 0, Disrespect: 1

URL: www.jstore.org

Shame literature citations: 0

Mtetwa, Sibongile et al. "You Are Wasting Our Drugs': Health Service Barriers to HIV Treatment for Sex Workers in Zimbabwe." BMC: Public Health. 2013. Web.

Although generally affected by HIV and regardless of the existence of proper health care services targeting specifically female sex workers, sex workers in Zimbabwe are refusing in persisting to seek antiretroviral treatment (ART). In this study, three focus group discussions, which served to explore their experiences and perceptions of seeking care, were transcribed and analyzed to identify any recurring themes related to interactions with health services in their decision-making process. At the end of their study, Mtetwa et al concluded these women resisted seeking available care due to several reasons: the fear of being humiliated, which reflected a broader stigma surrounding their work, competing time commitments or simply socio-economic difficulties. In order for sex workers' health to generally improve, possible actions include sensitizing health care workers through specialized training and providing opportunities for sex workers to collectively organize for treatment and rights. Abundant in the results, it appears women often refused to seek treatment or continue seeking treatment due to the hospital staff condescension, which in turn instantly sends a feeling of shame to the sex workers; in one instance, a nurse openly said to a 32 year old female sex worker participant "So, you are a prostitute and you actually have the guts to come here to waste our time and drugs on you..." Instances such as these occurred in of the participant's narratives during group discussions.

Shame: 2, Embarrassment: 4, Humiliation: 3, Disrespect: 2, (Stigma -9)

\section{http://www.biomedcentral.com/1471-2458/13/698/}

Shame literature citations: 0

4. Talebi, Miki, Kimberly Matheson, and Hymie Anisman. "Support, Depressive Symptoms, and the Stigma towards Seeking Mental Health Help: A Comparison of Asian Versus Euro- Caucasian Students." International Journal of Social Science Studies. 1.1 (2013): n. page. Web. www.redfame.com

Particularly the case for the younger population, a significant number of individuals affected by mental health issues will neglect to seek proper care for their symptoms; only $40 \%$ of this population will seek professional mental health services. Gathering 553 students to participate in the online study, of which 472 were Euro-Caucasian, factors taken into concern were depression symptoms and how it 
relates to social support and other-stigma/ self-stigma. That is, depression symptoms were looked for a correlation with social support, stigma caused by others and stigma caused by the participant themselves. It was hypothesized Asian students would be more prone to stigmatization and depressive symptoms would be more strongly associated with stigma in this group, as opposed to the Euro-Caucasian group. From the study, it was concluded the Asian student population did encounter greater stigmatization in seeking mental help and received less support from friends and parents. Focusing on the stigma of seeking professional mental help and correlation between depression symptoms, Talebi et al fail to make the shame literature connection as the emphasis of the article is placed on the stigma from seeking professional help and cultural differences, specifically in the differences of the Asian students, whose parents carry a perfectionist notion for their sons/ daughter to fulfill while in school.

Shame: 5

Embarrassment: 0

Humiliation: 0

Stigma: 122

Shame literature citations: 0

http://redfame.com/journal/index.php/ijsss/article/view/36/49

5. Severance, Laura et al. "The Psychological Structure of Aggression Across Cultures." Journal of Organizational Behavior. 34.6 (2013): 835865. Web.www.onlinelibrary.wiley.com

Looking into aggression across various cultures in the hopes of finding universal aggression dimensions, Severance et al uncovered the universal dimensions in all four countries were damages to self-worth, direct versus indirect aggression and physical versus verbal aggression; moreover, an infringement of personal resources dimension developed in the United States and Israel while the dimension of a degree of threat appeared in Pakistan. A universal phenomenon, Severance et al conclude by declaring this study confirms the conceptions of aggressive acts as, to some extent, universal. Although the study had several limitations, including the degree of aggression as a correlation to culture specific variables, this study further adds to the aggression literature in the anticipation to prevent and alleviate intercultural conflicts. Despite the fact that Severance et al mention shame in the article three times, it is clear they do not make the shame literature connection since shame is only mentioned as either a quote or a restatement of a referenced article; moreover, shame is touched over as a possible reason for aggression.

Shame: 3

Embarrassment: 1

Humiliation: 4

Disrespect: 12

http://onlinelibrary.wiley.com/doi/10.1002/job.1873/full

Shame literature citations: 1

In addition to the study of six subjects reported above, the following discussion will give a brief assessment of the many other fields of study which seem unaware of the connection to shame

Stigma, Social Pain and Suffering
Stigma has become the center of a huge body of research, but has not been defined in a way that is accepted by most researchers [19]. In the enormous number of stigma studies, very few even mention shame, much less define stigma as a type of shame.

In his frequently cited book on stigma [17], was a bold exception: he used the word continuously throughout. Here are four of his many sentences that contain the s-word. (I have bolded the shame terms. However, in the last quote, the word shameful was already bolded in the text). Notice that in the second quote, three shame terms occur in a single sentence:

Shame becomes a central possibility, arising from the individual's perception of one of his own attributes as being a defiling thing to possess, and one he can readily see himself as not possessing. (P. 7, first chapter.)

Most important of all, the very notion of shameful differences assumes a similarity in regard to crucial beliefs, those regarding identity. (P. 13)

(The stigmatized person's) identification with (other) offenders like himself (e.g. mentally ill) holds him to what repels him, transforming repulsion into shame, and then transforming ashamedness itself into something of which he is ashamed. (P.108)

Once the dynamics of shameful differentness are seen as a general feature of social life, one can go on to look at the relation of their study to the study of neighboring matters associated with the term "deviance"...(P. 140, last chapter).

Goffman does not define stigma as shame directly (as most dictionaries do), but his frequent and emphatic usage in these and other passages imply it.

Goffman's frequent usage suggests that stigma is a certain kind of shame, the kind caused by labeling. Many of the later books and articles on stigma cite Goffman's book, but avoid the shame word. One edited book (Heatherton et al. 2000) cites it 9 times, but without noting his insistent use of the S-word. The word itself is used 5 times in this book, but never alone; as frequently happens when it occurs, the taboo is softened because it is mixed in with other, less offensive or more abstract emotion names, such as guilt, anxiety, etc.

The idea of "the looking-glass self" [20] is cited in three of the Heatherton et al book's chapters. Indeed, it is used in the title of one of the chapters: "The Looking-glass Self Revisited." But none of the citations mention that Cooley proposed that the process of seeing one's self in the eyes of others always ends in pride or shame. The widely quoted review of the stigma literature [21] cites Goffman's book three times but doesn't cite Cooley nor mention shame at all.

The idea of social suffering and pain is much broader than that caused by labeling alone: it involves all pain caused by any kind of rejection $[1,22]$. Like the edited books on stigma, these two volumes hardly mention shame, the emotion that is caused by real or imagined rejection [10]. Shame does not appear in the index of either volume. It is used 9 times in the MacDonald and Jensen-Campbell book, but only in passing or in the company of other unrelated emotions.

Another field to be considered is what is called "the desire for status"[5]. Unlike the fields named above that seem to have no idea that their topic is intimately connected with shame, this field shows slight signs of recognition. The word shame occurs five times in the essay itself in the course of telling the findings of five of the articles that are cited, because these articles use the s-word. This is a very thin 
relationship to the idea of shame, since there is no citing of the shame literature itself.

Finally, there are a large number of studies of the outcomes of rejection, especially rejection that leads to violence.

Ignoring the whole shame literature surely doesn't help the attempts to understand stigma, social pain and suffering, status striving, rejection and violence, etc. Unfortunately, this practice does help the public continue to ignore the shame in their lives and in their societies. It would appear that social/behavioral and neurological scientists are just as loath to use the S-word as the general public, if not more so. My wife, Suzanne Retzinger, a hospice counselor, told me that the S-word is occasionally brought up in sessions by clients without her prompting. Perhaps we scientists need some counseling on this topic. What is to be done: study and discuss shame directly instead of hiding it behind various covers. This undertaking would require making some fundamental definitions since the vernacular words for shame and its opposite, pride, are confused and confusing.

From my experience with these problems, it seems to me that the emotion language and ideas in modern societies are not just tropes, but a special type of trope. An ordinary trope is a widespread belief held so firmly that it goes without saying [22]. The vast structure of beliefs in a society is made up of interlocking tropes, but some of them are strongly defended against change of any kind. Kepler's discovery that the planets revolve the sun rather than the earth probably wasn't a huge shock to the public; their daily life didn't depend on it. But the idea that some emotions are positive and others negative seems to be a crucial trope, one that will be defended as if peoples' lives depended on it, and will continue to face virtually immovable resistance to change [23-30].

\section{Conclusion}

A taboo on shame in modern societies is suggested by the large number of studies of shame that use alternative terms. There is probably much higher than the five to one that we found in our systematic study. The idea that shame is taboo in modern societies points to the necessity of bringing it out in the open. Perhaps it can be done first in scholarship, then with the public. It appears that many of the worse features of modern societies, such as withdrawal, violence and unnecessary conflict, may be caused, in part, by the hiding of shame. Other areas that might be better understood: the punitive element in legal systems, especially in imprisonment, hung negotiations and mediations, and individual and mass prejudice in social class, ethnic/racial and gender relationships. Perhaps it may be possible to bring shame out of the closet at least as far as been done with sex.

\section{References}

1. Kinsey, Alfred, W. Pomeroy, C Martin (1948) Sexual Behavior in the Human Male. Indiana University Press.

2. Masters WH, Johnson V E (1966) Human Sexual Response. New York: Bantam Books.

3. Gilligan James (1997) Violence - reflections on a national epidemic. New York: Vintage Books.
4. Icaboni Marco (2008) Mirroring People. New York: Farrar, Straus, and Giroux.

5. Anderson C, Hildreth J A D, Howland L (2015) Is the Desire for Status a Fundamental Human Motive? A Review of the Empirical Literature. Psychological Bulletin.

6. Dennis S (2006) Globalization: the Hidden Agenda. Cambridge: Polity.

7. Norbert E (1939) Uber den Prozess der Zivilisation, Reprinted in 1978 as The Civilizing Process. London: Blackwell.

8. David L (2009) The Role of Humiliation in Collective Political Violence. Sydney: U. of Sydney Press.

9. Neil W (2010) Familicidal Hearts: The Emotional Style of 211 Killers. Oxford: Oxford University Press.

10. Scheff T (1987) Case Study of an Interminable Quarrel, in The Role of Shame in Symptom Formation, Helen B. Lewis (Editor). Patterson, NJ: LEA.

11. Cheng J, Tracey J, The psychology of social status New York: Springer 159-178.

12. Kaufman, Gershon (1989) The Psychology of Shame. New York: Springer.

13. Louis G, Winget C, G Gleser (1969) Manual of Instruction for Using the Gottschalk-Gleser Content Analysis Scales. Berkeley: UC Press.

14. Retzinger, Suzanne (1995) Identifying Shame and Anger in Discourse. American Behavioral Science, 38: 104-113

15. Leape, Lucian L MD, Shore Miles F MD, Dienstag Jules L, MD; Mayer,et al.(2012) The Nature and Causes of Disrespectful Behavior by Physicians. Academic Medicine, 87: 845-852.

16. Silvan T (1963) Affect, Imagery, Consciousness. New York: Springer, 2.

17. Erving G (1983) Stigma. Englewood Cliffs: New Jersey.

18. Link, Bruce, Jo Phelan (2001.)Conceptualizing Stigma. Annual Review of Sociology 27: 363-385.

19. Scheff, T, David Fearon Jr (2004) Social and Emotional Components in Self-Esteem. Journal of the Theory of Social Behavior. 34: 73-90.

20. Cooley, Charles H (1964) Human Nature and the Social Order. New York: Schocken.

21. Brenda M, O'Brien LT (2005) The social psychology of stigma. Annual Review of Psychology, 56: 393-421.

22. Gibb SC (2015) Tropes and the generality of laws. The Problem of Universals in Contemporary Philosophy. Cambridge: Cambridge University Press.

23. Crosskey LB, Curry JF, Leary MR (2015) Role transgressions, shame, and guilt among clergy. Pastoral Psychology, 7-33.

24. Hetherton, Ted, Kleck, R, Hebl, M, Jull J The Social Psychology of Stigma. New York: Guilford.

25. Leary MR, Twenge JM, Quinlivan E (2006) Interpersonal rejection as a determinant of anger and aggression. Personality and Social Psychology Review, 10: 111-132.

26. Leary MR, Jongman-Sereno KP (2015) When rejection kills: The central role of low relational value in school violence. International Journal of Developmental Science 8: 25-27.

27. (2011) A Theory of Multiple Killing. Aggression and Violent Behavior, 16: 453-460.

28. (2014) Defining Stigma. International Journal of Social Psychiatry, 222-225.

29. (2015) Toward Defining Basic Emotions. Qualitative Inquiry, 21: 111-121.

30. Williams KD, Forgas JP, von Hippel B (2015) The social outcast: Ostracism, social exclusion, rejection, and bullying. New York: Cambridge University Press. 